\title{
Acceleration Analysis of 3-DOF Planar Parallel Manipulators by Means of Screw Theory
}

\author{
Soheil Zarkandi ${ }^{1^{*}}$ \\ ${ }^{1}$ Department of Mechanical Engineering, Babol Noshirvani University of Technology, Babol, Iran \\ Corresponding e-mail: zarkandi@gmail.com
}

\begin{abstract}
This paper deals with the second order kinematics of three degree-of-freedom (DOF) planar parallel manipulators. The simple and compact expressions are derived for both the inverse and forward acceleration analyses using screw theory. Moreover, as an example, a 3-DOF planar parallel manipulator is introduced and its kinematics is analyzed using the proposed method.
\end{abstract}

Keywords: planar parallel manipulators; velocity analysis; acceleration analysis; Screw theory

\section{INTRODUCTION}

As it is well known a general parallel manipulator is a mechanism composed of a mobile platform connected to the ground by several independent kinematic chains, called serial connector chains. Each serial connector chain can be regarded as a serial manipulator with both actuated and passive joints, the former providing the actuation to the mobile platform. Despite of a reduced workspace and a more complex solution of the direct kinematic problem than serial manipulators, the higher stiffness, accuracy and payload/weight ratio which can be achieved by parallel manipulators make them attractive systems for industrial applications.

A concept which has been employed extensively in kinematic analysis of parallel manipulators is screw theory. For instance, it has been used in identifying the degrees of freedom [1], singularity analysis [2], type synthesis [3] and also in velocity and acceleration analysis $[4,5]$ of spatial parallel manipulators. On the other hand, in the case of planar parallel manipulators, the literature is so limited $[6,7]$.

According to reciprocal screw theory [8-10], a screw $\$$ is defined by a straight line (Fig. 1) with an associated pitch $h$ and is conveniently denoted by a six dimensional vector:

$$
\$=\left[\begin{array}{l}
\mathbf{s} \\
\mathbf{t}
\end{array}\right]
$$

where $\mathbf{s}$ is a unit vector along the screw axis, $\mathbf{t}=\mathbf{r} \times \mathbf{s}$ $+h \mathbf{s}$ defines the moment of the screw axis about the origin of the reference frame and $\mathbf{r}$ is the position vector of any point on the screw axis with respect to the reference frame.

Manipulator joints can be modeled with their screw coordinates [10]. For revolute joints the associated pitch is equal to zero and for prismatic joints the associated pitch is equal to infinity which is normalized, yielding respectively

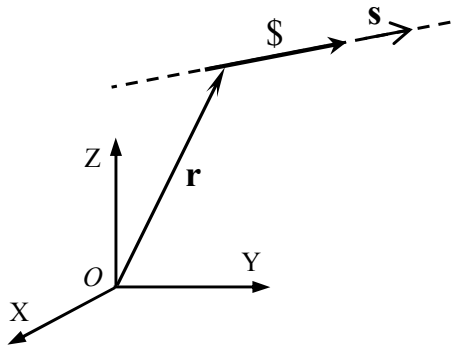

Figure 1. A screw [3].

$\$=\left[\begin{array}{c}\mathbf{s} \\ \mathbf{r} \times \mathbf{s}\end{array}\right]$ and $\$=\left[\begin{array}{l}\mathbf{0} \\ \mathbf{s}\end{array}\right]$

Physically, a screw can be used to represent the twist or wrench of a rigid body. For a twist $\mathbf{T}=\left[\boldsymbol{\omega}^{T} \mathbf{v}^{T}\right]^{T}, \boldsymbol{\omega}$ denotes the angular velocity and $\mathbf{v}$ denotes the linear velocity of a point in the rigid body. On the other hand, for a wrench $\mathbf{W}=\left[\mathbf{f}^{T} \mathbf{m}^{T}\right]^{T}, \mathbf{f}$ denotes the resultant force and $\mathbf{m}$ denotes the resultant moment about the origin of the reference frame.

For two screws $\$_{1}=\left[\begin{array}{ll}\mathbf{s}_{1} & \mathbf{t}_{1}\end{array}\right]^{T}$ and $\$_{2}=\left[\begin{array}{ll}\mathbf{s}_{2} & \mathbf{t}_{2}\end{array}\right]^{T}$, Lie product or dual motor product is defined as [11]

$\left[\begin{array}{ll}\$_{1} & \$_{2}\end{array}\right]=\left[\begin{array}{c}\mathbf{s}_{1} \times \mathbf{s}_{2} \\ \mathbf{s}_{1} \times \mathbf{t}_{2}-\mathbf{s}_{2} \times \mathbf{t}_{1}\end{array}\right]$

Moreover, the reciprocal product of two screws is defined as

$$
\$_{1} \circ \$_{2}=\mathbf{s}_{1} \cdot \mathbf{t}_{2}+\mathbf{s}_{2} \cdot \mathbf{t}_{1}
$$

when $\$_{1} \circ \$_{2}=0$ then two screws $\$_{1}$ and $\$_{2}$ are said to be reciprocal.

To define the concept of reciprocity for revolute and prismatic joints, assume a wrench is acting on a twist. If the line of action of a force crosses the axis of a revolute joint, the force does not affect the rotation 
of the joint, i.e., the force cannot exert any moment about the joint. Therefore, the force is reciprocal to the joint. Also, if the moment of the wrench is perpendicular to the revolute joint axis, the moment is reciprocal to the joint. If the line of action of a force is perpendicular to the direction of a prismatic joint, the force does not affect the translation of the joint. Also a moment, regardless of its direction, is reciprocal to a prismatic joint. Therefore, two screws are reciprocal if the power developed by the wrench on a twist is zero [7], i.e.,

$\boldsymbol{\omega} \cdot \mathbf{m}+\mathbf{v} \cdot \mathbf{f}=0$

The purpose of this paper is to show how screw theory can be used to systematically analyze the acceleration of 3-DOF planar parallel manipulators (PPMs). The proposed method can be considered as an extension of the results previously obtained by Rico and Duffy [4] in kinematic analysis of spatial parallel manipulators.

\section{KINEMATICS OF PLANAR PARALLEL MANIPULATORS}

Here, we are interested only in symmetrical 3-DOF fully-parallel planar manipulators; thus these manipulators have three legs while each of them is composed of two passive and one active joint (Fig. 2). To describe the various types of legs, a revolute joint is denoted by $R$ and a prismatic joint by $P$. When a joint is active (actuated), its corresponding symbol will be underlined.

For manipulators with $\delta$ DOFs, where $\delta<6$, such as planar manipulators, the same $6-\delta$ coordinates of the joint screws will be zero at any configuration [12]. Thus, for PPMs, we can use 3-entries screws to represent the joint screws. For these manipulators, kinematic screws associated with the revolute and prismatic joints will be respectively

$$
\$_{R}=\left[\begin{array}{c}
1 \\
-\mathbf{E r}_{R}
\end{array}\right] \text { and } \$_{P}=\left[\begin{array}{l}
0 \\
\mathbf{s}
\end{array}\right]
$$

where $\mathbf{r}_{R}$ is the $2 \mathrm{D}$ position vector of revolute joint center and $\mathbf{s}$ is a $2 \mathrm{D}$ unit vector along the direction of prismatic joint. Moreover, E is a $2 \times 2$ orthogonal matrix rotating vectors in a plane through an angle of $90^{\circ}$ counterclockwise, i.e.,

$$
\mathbf{E}=\left[\begin{array}{cc}
0 & -1 \\
1 & 0
\end{array}\right]
$$

The concepts of velocity and reduced acceleration states were first introduced by Sugimoto [11] and Rico and Duffy [4] respectively. These quantities can be redefined for PPMs as two 3-entries screws, as follows

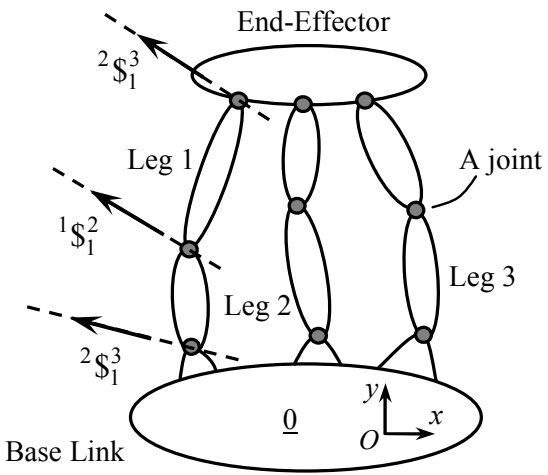

Figure 2. A typical 3-DOF planar parallel manipulator along with the screws of the first leg.

$\mathbf{V}_{O}=\left[\begin{array}{c}\omega \\ \tilde{\mathbf{v}}_{O}\end{array}\right]$

and

$\mathbf{A}_{O}=\left[\begin{array}{c}\dot{\omega} \\ \widetilde{\mathbf{a}}_{O}-\omega \mathbf{E} \widetilde{\mathbf{v}}_{O}\end{array}\right]$

where $\omega$ and $\dot{\omega}$ are the angular velocity and acceleration of end-effector respectively. In addition, $\widetilde{\mathbf{a}}_{0}$ and $\widetilde{\mathbf{v}}_{0}$ are $2 \mathrm{D}$ vectors representing translational velocity and acceleration of the point $O$, respectively.

In the following sections, 3 -entries screws are used to analyze the kinematics of planar parallel manipulators.

\section{VELOCITY ANALYSIS OF PPMS}

The velocity state of the end-effector of a parallel manipulator, body $\mathrm{m}$, with respect to the base link, body 0 , can be expressed as a linear combination of the infinitesimal screws representing kinematic pairs of each serial kinematic chain, see Sugimoto [11]. Using the 3-entries screws presented above, velocity state of the end-effector of a PPM can be obtained as

$$
\mathbf{V}_{O}={ }^{0} \dot{\theta}_{i}^{10} \$_{i}^{1}+\dot{\theta}_{i}^{21} \$_{i}^{2}+{ }^{2} \dot{\theta}_{i}^{32} \$_{i}^{3}, i=1,2,3
$$

where ${ }^{j} \dot{\theta}_{i}^{j+1}$ and ${ }^{j} \$_{i}^{j+1}$ are the generalizes velocity and the associated screw of the joint connecting links $j$ and $j+1$ in the $i$-th kinematic chain respectively (Fig. 2).

The inverse velocity analysis consists of finding joint rate velocities of the parallel manipulator, given the velocity state of end-effector with respect to fixed platform. According to Eq. (10), it follows that

$$
\mathbf{J}_{i}^{-1} \mathbf{V}_{O}=\dot{\boldsymbol{\theta}}_{i}, i=1,2,3
$$

where $\mathbf{J}_{i}=\left[\begin{array}{lll}{ }^{0} \$_{i}^{1} & { }^{1} \$_{i}^{2} & { }^{2} \$_{i}^{3}\end{array}\right]$ is the $i$-th Jacobian matrix of the corresponding leg and 
$\dot{\boldsymbol{\theta}}_{i}=\left[\begin{array}{lll}{ }^{0} \dot{\theta}_{i}^{1} & { }^{1} \dot{\theta}_{i}^{2} & { }^{2} \dot{\theta}_{i}^{3}\end{array}\right]^{T}$ is the $i$-th vector of joint rate velocities.

On the other hand, the forward velocity analysis consists of finding the velocity state of end-effector, with respect to the fixed platform, given the active joint rate velocities.

To eliminate the passive joint velocities from Eq. (10), it is multiplied (via the reciprocal screw product) with a screw, denoted by $\$_{i}^{r}$, reciprocal to all passive joint screws in the $i$-th leg. The reciprocal screw depends on the two passive joints screws in the $i$-th leg. If the two passive joints are revolute, the reciprocal screw is a zero-pitch screw (i.e., a pure force) with an axis lying in the $x y$ plane and intersecting the centers of the revolute passive joints (Fig. 3a). On the other hand, when one of the passive joints is revolute and the other is prismatic, the reciprocal screw is a pure force passing through the $R$ joint center and perpendicular to the direction of the $P$ joint $^{1}$ (Fig. 3b) [6]. In the both cases, $\$_{i}^{r}$ can be expressed as

$$
\$_{i}^{r}=\left[\begin{array}{c}
\mathbf{e} \\
m
\end{array}\right]
$$

where $\mathbf{e}$ is a $2 \mathrm{D}$ unit vector along the direction of the reciprocal screw and $m$ is the magnitude of the moment of $\mathbf{e}$ with respect to the origin of the reference coordinate frame.

Reciprocal product of both sides of Eq. (10) with $\$_{i}^{r}$ leads to

$$
\Delta \$_{i}^{r} \mathbf{V}_{O}=\left(\Delta \$_{i}^{r}\right)^{T} \$_{i}^{a} \dot{\theta}_{i}^{a}, i=1,2,3
$$

in which the superscript " $a$ " denotes the active joint and $\Delta$ is given by

$$
\Delta=\left[\begin{array}{ccc}
0 & 0 & 1 \\
1 & 0 & 0 \\
0 & 1 & 0
\end{array}\right]_{3 \times 3}
$$

Upon writing Eq. (13) for $i=1,2,3$, we obtain

$$
\mathbf{K} \mathbf{V}_{O}=\mathbf{M} \dot{\boldsymbol{\theta}}
$$

where $\dot{\boldsymbol{\theta}}=\left[\begin{array}{lll}\dot{\theta}_{1}^{a} & \dot{\theta}_{2}^{a} & \dot{\theta}_{3}^{a}\end{array}\right]^{T}$ is the vector of active joint velocities. $\mathbf{K}$ and $\mathbf{M}$ are the Jacobian matrices of

${ }^{1}$ For a passive joint of the revolute or prismatic type, the corresponding screw will be generally of the form: $\$=[00 p$ $\left.\begin{array}{lll}q & r & 0\end{array}\right]^{T}$ where $p, q$ and $r$ are the non zero entries and the reciprocal screw can be $\$^{r}=\left[\begin{array}{llllll}e_{1} & e_{2} & 0 & 0 & 0 & \mathrm{~m}\end{array}\right]^{T}$. Thus, According to the physical meaning of the reciprocal screws, $\$^{r}$ denotes a pure force lying in the $x y$ plane. the manipulator and obtained as

$$
\mathbf{K}=\left[\begin{array}{c}
\left(\Delta \$_{1}^{r}\right)^{T} \\
\left(\Delta \$_{2}^{r}\right)^{T} \\
\left(\Delta \$_{3}^{r}\right)^{T}
\end{array}\right]_{3 \times 3}
$$

and

$$
\mathbf{M}=\left[\begin{array}{ccc}
\left(\Delta \$_{1}^{r}\right)^{T} \$_{1}^{a} & 0 & 0 \\
0 & \left(\Delta \$_{2}^{r}\right)^{T} \$_{2}^{a} & 0 \\
0 & 0 & \left(\Delta \$_{3}^{r}\right)^{T} \$_{3}^{a}
\end{array}\right]_{3 \times 3}
$$

Therefore, velocity state of the end-effector can be gained as

$$
\mathbf{V}_{O}=\mathbf{K}^{-1}(\mathbf{M} \dot{\boldsymbol{\theta}})
$$

Finally, once the angular velocity of end-effector and translational velocity of the reference point $O$ are calculated, the translational velocity of operating point of the end-effector, $E$, is calculated using classical kinematics. Indeed

$$
\widetilde{\mathbf{v}}_{E}=\widetilde{\mathbf{v}}_{O}+\omega \mathbf{E} \mathbf{r}_{E / O}
$$

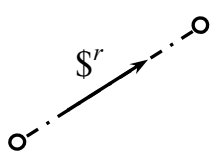

(a)

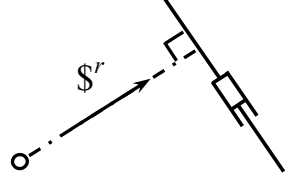

(b)
Figure 3. Reciprocal screw for (a) two passive R joints and (b) one passive $\mathrm{R}$ joint and one passive $\mathrm{P}$ joint [6].

\section{ACCELERATION ANALYSIS OF PPMS}

Rico and Duffy [13] obtained an expression for the reduced acceleration state of the end-effector of a serial chain in terms of joint screws. This expression can be redefined for PPMs using the 3-entries screws as

$$
\mathbf{A}_{O}={ }^{0} \ddot{\theta}_{i}^{10} \$_{i}^{1}+{ }^{1} \ddot{\theta}_{i}^{21} \$_{i}^{2}+{ }^{2} \ddot{\theta}_{i}^{32} \$_{i}^{3}+\$_{\text {Lie }}^{i}, i=1,2,3
$$

where ${ }^{j} \ddot{\theta}_{i}^{j+1}$ is the generalized acceleration of the joint connecting links $j$ and $j+1$ in the $i$-th kinematic chain and $\$_{\text {Lie }}^{i}$ is defined as

$$
\$_{\text {Lie }}^{i}=\left[{ }^{0} \dot{\theta}_{i}^{10} \$_{i}^{1}{ }^{1} \dot{\theta}_{i}^{21} \$_{i}^{2}+{ }^{2} \dot{\theta}_{i}^{32} \$_{i}^{3}\right]+\left[{ }^{1} \dot{\theta}_{i}^{21} \$_{i}^{2} \quad{ }^{2} \dot{\theta}_{i}^{32} \$_{i}^{3}\right]
$$

In Eq. (19), the rate of passive joints can be obtained by dot multiplying both sides of Eq. (10) by a vector perpendicular to the screw vectors of the other two joints. For instance, if ${ }^{1} \dot{\theta}_{i}^{2}$ denotes the rate of a passive joint then 


$$
{ }^{1} \dot{\theta}_{i}^{2}=\frac{\left({ }^{0} \$_{i}^{1} x^{2} \$_{i}^{3}\right)^{T} \mathbf{V}_{O}}{\left({ }^{0} \$_{i}^{1} \times{ }^{2} \$_{i}^{3}\right)^{T}\left({ }^{1} \$_{i}^{2}\right)}
$$

In addition, if $\$_{1}=\left[\begin{array}{lll}p_{1} & q_{1} & r_{1}\end{array}\right]^{T}$ and $\$_{2}=\left[\begin{array}{lll}p_{2} & q_{2} & r_{2}\end{array}\right]^{T}$ are two planar screws of the manipulator joints then Lie product of these two screws can be obtained as (see appendix A)

$$
\left[\begin{array}{ll}
\$_{1} & \$_{2}
\end{array}\right]=\left[\begin{array}{lll}
0 & -p_{1} r_{2}-p_{2} r_{1} & p_{1} q_{2}+p_{2} q_{1}
\end{array}\right]^{T}
$$

Now, the inverse and forward acceleration analyses of the PPMs can be carried out using the above relations.

Following the trend of previous section, the inverse acceleration analysis, in other words computation of the joint rate accelerations of the manipulator given the acceleration state of the end-effector, can be calculated, according to Eq. (18), as follows

$$
\mathbf{J}_{i}^{-1}\left(\mathbf{A}_{O}-\$_{L i e}\right)=\ddot{\boldsymbol{\theta}}_{i}
$$

where $\ddot{\boldsymbol{\theta}}_{i}=\left[\begin{array}{lll}{ }^{0} \ddot{\theta}_{i}^{1} & { }^{1} \ddot{\theta}_{i}^{2} & { }^{2} \ddot{\theta}_{i}^{3}\end{array}\right]^{T}$. Whereas the forward acceleration analysis, in other words the computation of the accelerator of the end-effector with respect to the base given the joint rate accelerations of the actuated prismatic joints, are calculated by means of the expression

$$
\mathbf{K A} \mathbf{A}_{O}=\mathbf{M} \ddot{\boldsymbol{\theta}}+\mathbf{b}
$$

in which $\ddot{\boldsymbol{\theta}}=\left[\begin{array}{lll}\ddot{\theta}_{1}^{a} & \ddot{\theta}_{2}^{a} & \ddot{\theta}_{3}^{a}\end{array}\right]^{T}$ and vector $\mathbf{b}$ is obtained as follows

$$
\mathbf{b}=\left[\begin{array}{c}
\left(\$_{1}^{r}\right)^{T} \$_{L i e}^{1} \\
\left(\$_{2}^{r}\right)^{T} \$_{L i e}^{2} \\
\left(\$_{3}^{r}\right)^{T} \$_{L i e}^{3}
\end{array}\right]_{3 \times 1}
$$

Thus

$$
\mathbf{A}_{O}=\mathbf{K}^{-1}(\mathbf{M} \ddot{\boldsymbol{\theta}}+\mathbf{b})
$$

Finally, translational acceleration of point $E$ is computed using the following relation.

$$
\widetilde{\mathbf{a}}_{E}=\widetilde{\mathbf{a}}_{O}-\omega^{2} r_{E / O}+\dot{\omega} \mathbf{E} \mathbf{r}_{E / O}
$$

in which $r_{E / O}=\left|\mathbf{r}_{E / O}\right|$.

\section{ILLUSTRATIVE EXAMPLE}

One of the 3-DOF PPMs, proposed in the literature, is the 3-PRP star-triangle PPM [14] in which the base and moving platform (end-effector) of the manipulator are triangle- and star-shaped, respectively. Here, a new 3-PRP PPM is introduced by replacing the base and moving platform of the mentioned manipulator. Then, the kinematics of this new manipulator, which is called Triangle-Star PPM (Fig. 4), is analyzed using the results obtained above.

It is obvious that for analyzing the velocity and acceleration of a manipulator, the forward position analysis is an essential step. For the planar parallel manipulators, Hunt showed that the forward position kinematics admits at most 6 solutions [15] and several authors have shown independently that their forward position kinematics can be reduced as the solution of a characteristic polynomial of degree 6 , see for instance [16].

As shown in Fig. 4, the 3-PRP Triangle-Star PPM consists of a fixed star and a moving triangle (endeffector) connected to each other by three legs. Each leg starts with a prismatic actuator followed by two passive revolute and prismatic joints. Fig. 5 shows the Geometric model of the manipulator along with the notations used to define the geometric parameters. The reference frame $\{\mathrm{X}, \mathrm{Y}\}$ is attached at the centered point $O$ of the fixed star. $\rho_{i}$ is the actuated joint variable of the $i$-th leg. Vertex $B_{1}$ of the moving triangle is chosen as the operating point. Therefore, the output variables are the position coordinates $(x, y)$ of point $B_{1}$ and $\phi$ that is the orientation angle of endeffector.

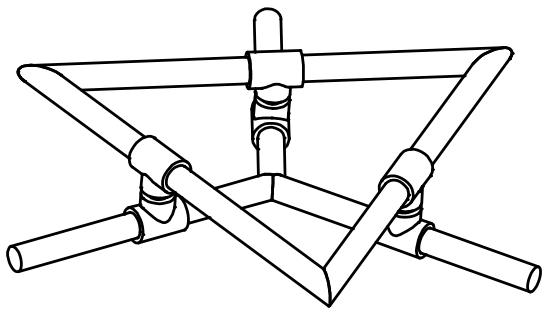

Figure 4. The Triangle-Star planar parallel manipulator.

With reference to Fig. 5, three sets of constraint equations can be written through three legs of the manipulator as follows

$$
\begin{aligned}
& \left\{\begin{array}{l}
y-\overline{A_{1} B_{1}} \sin \phi-y_{A 1}=0 \\
x-\overline{A_{1} B_{1}} \cos \phi-x_{A 1}=0
\end{array}\right. \\
& \left\{\begin{array}{l}
y+\overline{A_{2} B_{1}} \sin (\beta-\phi)-y_{A 2}=0 \\
x-\overline{A_{2} B_{1}} \cos (\beta-\phi)-x_{A 2}=0
\end{array}\right. \\
& \left\{\begin{array}{l}
y-\overline{B_{1} B_{3}} \sin \phi+\overline{A_{3} B_{3}} \sin (\alpha+\phi)-y_{A 3}=0 \\
x-\overline{B_{1} B_{3}} \cos \phi+\overline{A_{3} B_{3}} \cos (\alpha+\phi)-x_{A 3}=0
\end{array}\right.
\end{aligned}
$$

in which the parameters $x_{A i}$ and $y_{A i}$ can be obtained by

$$
\begin{aligned}
& x_{A i}=\left(R-\rho_{i}\right) \cos \gamma_{i} \\
& y_{A i}=\left(R-\rho_{i}\right) \sin \gamma_{i}
\end{aligned}
$$




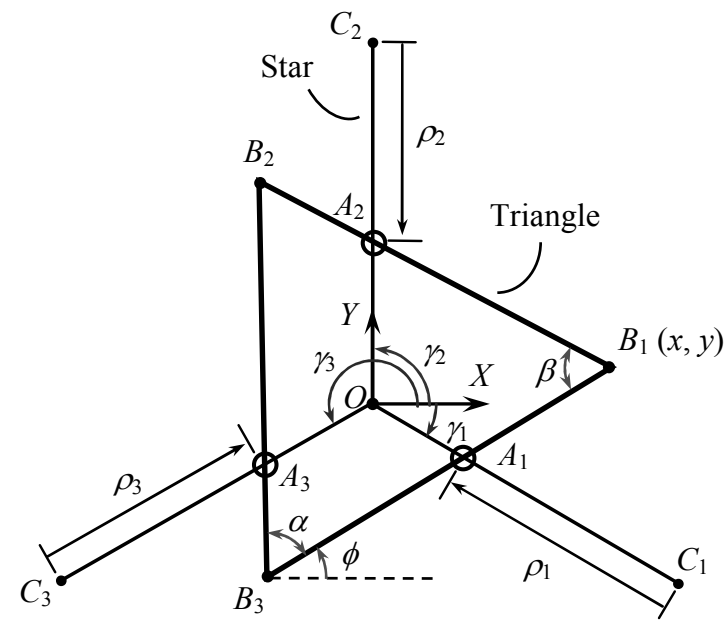

Figure 5. Geometric model of the triangle-star planar parallel manipulator.

where $R=\overline{O C_{i}} \quad(i=1,2,3)$ and $\gamma_{i}$ is the angle between the corresponding branch of the fixed star and the $x$-axis.

Obtaining $A_{i} B_{i}(i=1,2,3)$ from the first equation in each set of Eqs. (26)-(28) and introducing it into the second equation of the same set leads to

$$
\begin{aligned}
& Z_{1} x+Z_{2} y=Z_{3} \\
& Z_{4} x+Z_{5} y=Z_{6} \\
& Z_{7} x+Z_{8} y=Z_{9}
\end{aligned}
$$

where

$$
\begin{aligned}
& Z_{1}=\sin \phi \\
& Z_{2}=-\cos \phi \\
& Z_{3}=y_{A 1} \cos \phi-x_{A 1} \sin \phi \\
& Z_{4}=\sin (\beta-\phi) \\
& Z_{5}=\cos (\beta-\phi) \\
& Z_{6}=-y_{A 2} \cos (\beta-\phi)-x_{A 2} \sin (\beta-\phi) \\
& Z_{7}=\sin (\alpha+\phi) \\
& Z_{8}=-\cos (\alpha+\phi) \\
& Z_{9}=-\overline{B_{1} B_{3}} \sin \alpha+y_{A 3} \cos (\alpha+\phi)-x_{A 3} \sin (\alpha+\phi)
\end{aligned}
$$

Eqs. (31) and (32) constitute a system of two linear equations in two unknowns $x$ and $y$, thus these two variables can be computed using Cramer's rule as

$$
x=\frac{Z_{6} Z_{8}-Z_{5} Z_{9}}{Z_{4} Z_{8}-Z_{5} Z_{7}}, y=\frac{Z_{4} Z_{9}-Z_{6} Z_{7}}{Z_{4} Z_{8}-Z_{5} Z_{7}}
$$

Now by introducing Eq. (33) into Eq. (30) and using the trigonometric entities:

$$
\sin \phi=\frac{2 u}{1+u^{2}} \text { and } \cos \phi=\frac{1-u^{2}}{1+u^{2}} \quad\left(u=\tan \left(\frac{\phi}{2}\right)\right)
$$

a quadratic equation in $u$ is obtained as follows

$$
G_{2} u^{2}+G_{1} u+G_{0}=0
$$

while $G_{i}$ depends on the kinematic parameters of the manipulator and are presented in Appendix $B$. What is important to point out here is that the above equation admits two real solutions for $u$. Once $u$ is found, a unique value of the variables $\phi, x$ and $y$ can be calculated from Eqs. (34) and (33) ${ }^{2}$.

Now the forward position kinematics of the manipulator is solved with the following values (using SI units):

$$
\begin{aligned}
& \theta_{1}=-\frac{\pi}{6}, \theta_{2}=\frac{\pi}{2}, \theta_{3}=\frac{7 \pi}{6}, R=80, \alpha=\beta=\frac{\pi}{3}, \\
& \overline{B_{i} B_{i+1}}=114(i=1,2,3)
\end{aligned}
$$

Further, it is chosen that the instantaneous generalized coordinates are governed by the periodical functions:

$$
\begin{aligned}
& \rho_{1}=26-10 \sin (t) \\
& \rho_{2}=47+10 \sin (t) \\
& \rho_{3}=59-10 \sin (t) \cos (t), t=0, \ldots, 2 \pi
\end{aligned}
$$

Thus, moving triangle begins its motion at the time $t=0$ and $2 \pi$ seconds later returns to its original pose. With the above data, coefficients $G_{i}(i=0,1,2)$ are calculated for the initial configuration of manipulator $(t=0)$ which are listed in Table 1. Then solving Eq. (35) yields two real solutions for $u$ which are

2 It is worth noting that, in contrast to the proposed manipulator, the forward position problem of the Star-Triangle PPM has only one solution [14]. 
presented in Table 2 along with the corresponding values of $\phi, x$ and $y$.

Finally, by calculating the parameters $\$_{i}^{a}, \$_{i}^{r}, \dot{\rho}_{i}$, $\ddot{\rho}_{i}(i=1,2,3)$ and rate of the passive joints (Eq. (20)) and introducing them into Eqs. (16) and (24) while solution 1 of Table 2 is taken as the initial configuration, the most representative numerical results are obtained for forward velocity and acceleration analyses of the manipulator that are shown graphically in Figs. 6-8.

(a)

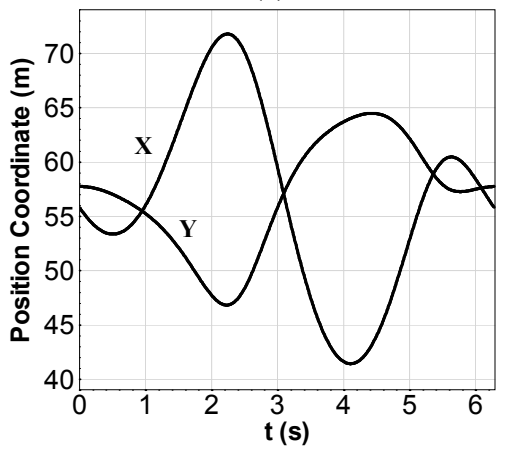

(b)

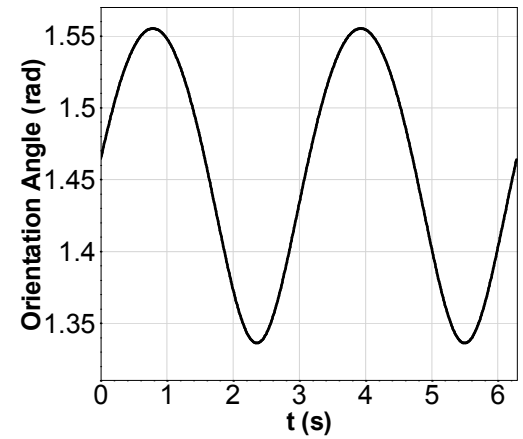

Figure 6. Time history of (a) position coordinates of the operating point of the end-effector, (b) the orientation angle of the end-effector.

Table 1 Coefficients $G i(i=0,1,2)$ obtained for the numerical example.

\begin{tabular}{cccc}
\hline Coefficient & value & Coefficient & value \\
\hline$G_{2}$ & 132.265372 & $G_{0}$ & 38.7346282 \\
$G_{1}$ & -162 & & \\
\hline
\end{tabular}

Table 2 two real solutions obtained for the forward position kinematics of triangle-star PPM.

\begin{tabular}{cccc}
\hline No. & $u$ & $\phi$ & $(x, y)$ \\
\hline 1 & 0.899084 & 1.46461754 & $(55.797,57.745)$ \\
2 & 0.325726 & 0.62977756 & $(80.257,-2.592)$ \\
\hline
\end{tabular}

\section{CONCLUSION}

In this work, acceleration analysis of 3-DOFs planar parallel manipulators has been successfully approached by means of screw theory. To this end, the velocity and reduced acceleration states of end-effector

(a)

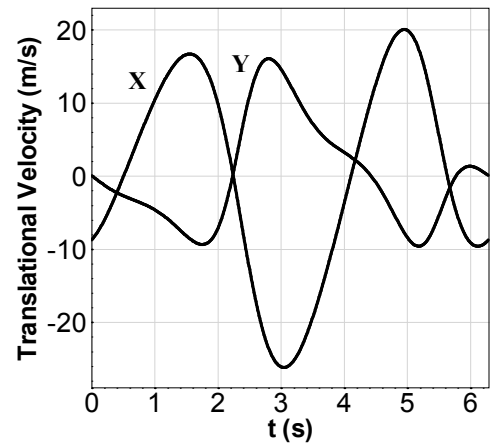

(b)

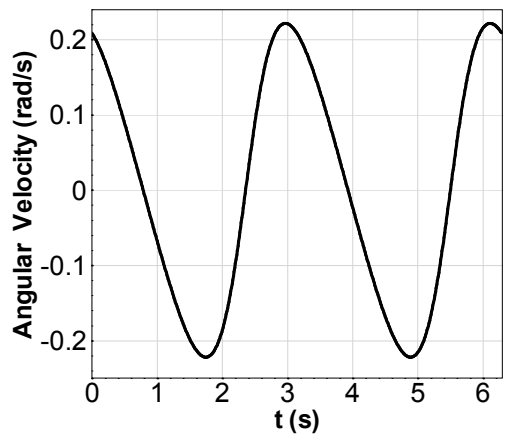

Figure 7. Time history of (a) the translational velocity of the operating point of the end-effector (b) the angular velocity of the end-effector.

(a)

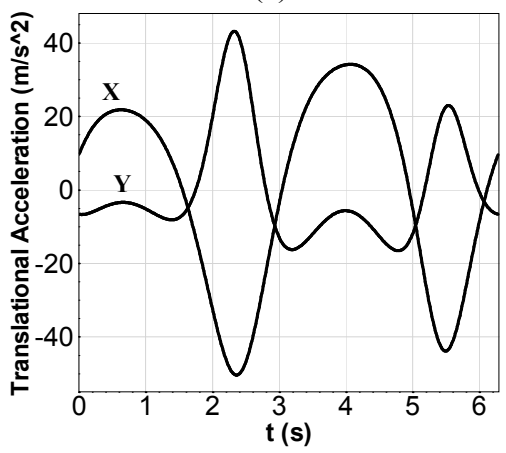

(b)

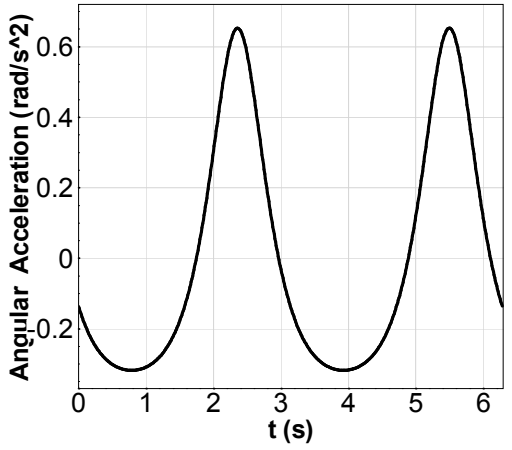

Figure 8. Time history of (a) the translational acceleration of the operating point of the end-effector 
(b) the angular acceleration of the end-effector. are written with respect to the reference frame in screw form through each one of the three legs of the manipulator. The obtained expressions are simple, compact and can be easily translated into computer codes. Finally, in order to show the efficiency of the presented methodology, it was applied on a 3-DOFs PPM known as triangle - star PPM. After solving the forward position kinematics of the manipulator, the forward velocity and acceleration kinematics were analyzed and shown graphically.

\section{REFERENCES}

[1] J.-S. Zhao, K. Zhou, Z.-J. Feng, 2004, "A theory of degrees of freedom for mechanisms", Mechanism and Machine Theory Vol. 39, No. 6, pp. 621-643.

[2] J.-S. Zhao, Z.-J. Feng, K. Zhou, J.-X. Dong, 2005, "Analysis of the singularity of spatial parallel manipulator with terminal constraints", Mechanism and Machine Theory Vol. 40, No. 3, pp. 275-284.

[3] X. Kong and C. M. Gosselin, 2004, "Type Synthesis of 3T1R 4-DOF Parallel Manipulators Based on Screw Theory", IEEE Transactions on Robotics and Automation, Vol. 20, No. 2, pp. 181-190.

[4] J. M. Rico Martınez, J. Duffy, 1998, "Forward and Inverse Acceleration Analyses of In-Parallel Manipulators", ASME J. Mech. Des. Vol. 122, No. 3, pp. 299-303.

[5] J. Gallardo-Alvarado, M. A. Garcia-Murillo and E. Castillo-Castaneda, 2013, “A 2(3-RRPS) parallel manipulator inspired by Gough-Stewart platform", Robotica, Vol. 31, pp. 381-388.

[6] I. A. Bonev, D. Zlatanov, C. M. Gosselin, 2003, "Singularity Analysis of 3-DOF Planar Parallel Mechanisms via Screw Theory", ASME J. Mech. Des. Vol. 125, pp. 254-258.

[7] K. H. Hunt, 1978, "Kinematic Geometry of Mechanisms, Oxford University Press, Oxford,.

[8] J. Phillips, 1984, "Freedom in machinery Introducing Screw Theory", Vol. 1, Cambridge University Press, Cambridge.

[9] J. Phillips, 1990, "Freedom in machinery - Screw Theory Exemplified", Vol. 2, Cambridge University Press, Cambridge.

[10] L. W. Tsai, 1999, "Robot Analysis, The Mechanics of Serial and Parallel Manipulators", Wiley-Interscience, Toronto, ON, Canada.

[11] K. Sugimoto, 1987, "Kinematic and dynamic analysis of parallel manipulators by means of motor algebra", ASME Journal of Mechanisms, Transmissions, and Automation in Design, Vol. 109, No. (1) pp. 3-7.

[12] D. Zlatanov, R. G. Fenton, and B. Benhabib, 1994, "Analysis of the Instantaneous Kinematics and Singular Configurations of Hybrid-Chain Manipulators", Proc. ASME 23rd Biennial Mechanisms Conference, DE-Vol. 72, Minneapolis, MN, pp. 467-476.
[13] J. M. Rico, J. Duffy, 1996, “An application of Screw algebra to the acceleration analysis of serial chains", Mechanism and Machine Theory, Vol. 31, No. 4, pp. 445-457.

[14] S. Zarkandi, 2011, "Kinematics of a Star-Triangle Planar Parallel Manipulator", Journal of Mechanical Science and Technology, Vol. 25, No. 12, pp. 3223-3230.

[15] K. H. Hunt, 1983, "Structural kinematics of in-parallel actuated robot arms", J. of Mechanisms, Transmissions and Automation in Design, Vol. 105, No. 4, pp. 705-712.

[16] C. Gosselin, J. Sefrioui and M. J. Richard, 1992, "Solutions polynomiales au problème de la cinématique des manipulateurs parallèles plans à trois degrés de liberté", Mechanism and Machine Theory, Vol. 27, No. 2, pp. 107-119.

\section{APPENDIX A}

If we write screws $\$_{1}$ and $\$_{2}$ in complete form, then we have

$$
\begin{aligned}
& \$_{1}=\left[\begin{array}{llllll}
0 & 0 & p_{1} & q_{1} & r_{1} & 0
\end{array}\right]^{T} \\
& \$_{2}=\left[\begin{array}{llllll}
0 & 0 & p_{2} & q_{2} & r_{2} & 0
\end{array}\right]^{T}
\end{aligned}
$$

Using the above screws, the terms on the right side of Eq. (3) can be obtained as

$$
\begin{aligned}
& \mathbf{s}_{1} \times \mathbf{s}_{2}=\mathbf{0} \\
& \mathbf{s}_{1} \times \mathbf{t}_{2}=\left[\begin{array}{lll}
-p_{1} r_{2} & p_{1} q_{2} & 0
\end{array}\right]^{T} \\
& \mathbf{s}_{2} \times \mathbf{t}_{1}=\left[\begin{array}{lll}
-p_{2} r_{1} & p_{2} q_{1} & 0
\end{array}\right]^{T}
\end{aligned}
$$

Introducing Eqs. (A1) to (A3) into Eq. (3) leads to the Eq. (21).

\section{APPENDIX B}

Coefficients $G_{i}(i=0,1,2)$

$$
\begin{aligned}
& G_{2}=\overline{B_{1} B_{3}} \sin \beta \sin \alpha-x_{A 3} \sin \beta \sin \alpha+y_{A 2} \cos \beta \sin \alpha- \\
& y_{A 1} \cos \alpha \sin \beta+x_{A 2} \sin \beta \sin \alpha- \\
& y_{A 1} \cos \beta \sin \alpha+y_{A 3} \cos \alpha \sin \beta \\
& G_{1}=-2 y_{A 2} \sin \alpha \sin \beta-2 x_{A 1} \cos \alpha \sin \beta+ \\
& 2 x_{A 3} \cos \alpha \sin \beta-2 x_{A 1} \cos \beta \sin \alpha+ \\
& 2 y_{A 3} \sin \alpha \sin \beta+2 x_{A 2} \sin \alpha \cos \beta \\
& G_{0}=\overline{B_{1} B_{3}} \sin \beta \sin \alpha-y_{A 3} \cos \alpha \sin \beta+x_{A 3} \sin \beta \sin \alpha- \\
& y_{A 2} \cos \beta \sin \alpha+y_{A 1} \cos \alpha \sin \beta+y_{A 1} \cos \beta \sin \alpha- \\
& x_{A 2} \sin \beta \sin \alpha
\end{aligned}
$$

